\title{
Selective Targeting of Angiopoietin-like 3 (ANGPTL3) with Vupanorsen for the Treatment of Patients with Familial Partial Lipodystrophy (FPLD): Results of a Proof-of-Concept Study
}

\author{
Maria Cristina Foss-Freitas \\ University of Michigan

\section{Baris Akinci} \\ University of Michigan

\section{Adam Neidert} \\ University of Michigan

\section{Victoria J Bartlett} \\ Akcea Therapeutics

\section{Eunju Hurh} \\ Akcea Therapeutics
}

\section{Ewa Karwatowska-Prokopczuk}

Akcea Therapeutics

Elif Oral ( $\square$ eliforal@med.umich.edu )

University of Michigan

\section{Research Article}

Keywords: Familial partial lipodystrophy, Angiopoietin-like protein 3, Triglycerides, Mixed meal test, Adipose tissue insulin resistance, Vupanorsen.

Posted Date: August 3rd, 2021

DOl: https://doi.org/10.21203/rs.3.rs-763069/v1

License: (c) (1) This work is licensed under a Creative Commons Attribution 4.0 International License.

Read Full License

Version of Record: A version of this preprint was published at Lipids in Health and Disease on December 1st, 2021. See the published version at https://doi.org/10.1186/s12944-021-01589-4. 


\section{Abstract}

Background: Familial partial lipodystrophy (FPLD) is a rare disease characterized by selective loss of peripheral subcutaneous fat associated with dyslipidemia and diabetes mellitus. Reductions in circulating levels of ANGPTL3 are associated with lower triglyceride and other atherogenic lipids, making it an attractive target for treatment of FPLD patients. This proof-of-concept study was conducted to assess the efficacy and safety of targeting ANGPTL3 with vupanorsen in patients with FPLD.

Methods: This was an open-label study. Four patients with FPLD (two with pathogenic variants in LMNA gene, and two with no causative genetic variant), diabetes ( $\mathrm{HbA} 1 \mathrm{c} \geq 7.0 \%$ and $\leq 12 \%$ ), hypertriglyceridemia ( $\geq 500 \mathrm{mg} / \mathrm{dL}$ ), and hepatic steatosis (hepatic fat fraction, HFF $\geq 6.4 \%$ ) were included. Patients received vupanorsen subcutaneously at a dose of $20 \mathrm{mg}$ weekly for 26 weeks. The primary endpoint was the percent change from baseline in fasting triglycerides at Week 27 . Other endpoints analyzed at the same time point included changes in ANGPTL3, fasting lipids and lipoproteins, insulin secretion/sensitivity, postprandial lipid and glycemic changes in response to a mixed meal test, HFF measured by MRI, and body composition measured by dual energy absorptiometry (DEXA).

Results: Baseline mean \pm SD fasting triglyceride level was $9.24 \pm 4.9 \mathrm{mmol} / \mathrm{L}(817.8 \pm 431.9 \mathrm{mg} / \mathrm{dL})$. Treatment resulted in reduction in fasting levels of triglyceride by $59.9 \%$, ANGPTL3 by $54.7 \%$, and in several other lipoproteins/lipids including very low- density lipoprotein cholesterol by $53.5 \%$, non-highdensity lipoprotein cholesterol by $20.9 \%$, and free fatty acids (FFA) by $41.7 \%$. The area under the curve for postprandial triglycerides, FFA, and glucose were reduced by $60 \%, 32 \%$, and $14 \%$, respectively. Treatment with vupanorsen also resulted in $55 \%$ reduction in adipose tissue insulin resistance index, while other insulin sensitivity indices and HbA1c levels were not changed. Additional investigations in HFF and in DEXA parameters suggested dynamic changes in fat partitioning during treatment. Adverse events observed were related to common serious complications associated with diabetes and FPLD, vupanorsen was well tolerated, and there was no effect on platelet count.

Conclusions: Although limited, these results suggest that targeting ANGPTL3 with vupanorsen could address several metabolic abnormalities in patients with FPLD.

\section{Clinical Trial: NCT03514420}

\section{Background}

Familial partial lipodystrophy (FPLD) is a rare disease characterized by selective loss of peripheral subcutaneous adipose tissue and redistribution, usually affecting the trunk and limbs, but preservation in other areas such as the face and neck [1]. It is usually associated with metabolic complications, including severe hypertriglyceridemia, hepatic steatosis, insulin resistance, diabetes mellitus and relatively low leptin levels [2]. Currently, there are no approved specific therapies for this disease in the US [3, 4]. The disease is heterogeneous both in presentation and etiology. There are numerous genes that cause the phenotype as well as polygenic forms. One can view FPLD as an extreme form of metabolic syndrome 
and Type 2 diabetes (T2DM) associated with truncal obesity, but the residual fat is likely highly dysfunctional and has more abnormalities due to the inherent genetic defects. In addition, patients present with a wide array of multi-system abnormalities and carry a high disease burden [5]. Accumulated evidence also suggests that this high disease burden may be associated with increased early mortality predominantly due to cardiovascular causes [6].

Angiopoietin-like protein 3 (ANGPTL3) is a glycoprotein secreted by the liver that inhibits lipoprotein lipase and endothelial lipase activity, two key enzymes involved in the metabolism of triglyceride-rich lipoproteins (TRLs) and high-density lipoprotein (HDL), respectively [7]. Individuals with homozygous loss-of-function mutations in the ANGPTL3 gene present with phenotype of familial combined hypolipidemia Type 2 (FHBL2), characterized by decreased plasma levels of triglycerides, low-density lipoprotein cholesterol (LDL-C), and high-density lipoprotein cholesterol (HDL-C). In addition, they have a reduced risk of coronary artery disease (CAD) $[8,9]$. Other beneficial metabolic effects that have been observed include increased insulin sensitivity and reductions in circulating free fatty acid (FFA) levels [10]. A similar hypolipidemic profile has been achieved by treatment with antisense oligonucleotides (ASO) targeting ANGPTL3 mRNA in the liver [11] or a monoclonal antibody against ANGPTL3 [9]. Therefore, we hypothesized that the reduction of ANGPTL3 levels had the potential to reduce hypertriglyceridemia and associated metabolic abnormalities in FPLD patients.

Vupanorsen is a second-generation $\mathrm{N}$-acetyl galactosamine (GalNAc3)-conjugated ASO targeting hepatic ANGPTL3 mRNA and represents a newer ASO technology approach. By having a high affinity for the hepatocyte-specific asialoglycoprotein receptor, vupanorsen provides therapeutic efficacy similar to that of parent unconjugated compound but with 20-fold lower dosing, thus reducing systemic exposure [11].

Given the lack of therapeutic options for patients with FPLD, we sought to determine whether vupanorsen had the potential to address multiple metabolic abnormalities in this disease. Therefore, this proof-ofconcept study in patients with FPLD who displayed hypertriglyceridemia, diabetes mellitus and hepatic steatosis was designed to evaluate the effect of vupanorsen on lipid metabolism, glucose control, insulin resistance, and body fat distribution, including hepatic fat content. The study also assessed safety and tolerability of vupanorsen in FPLD patients.

\section{Methods}

\section{Study design and patient population}

This proof-of-concept, phase 2, open-label study enrolled 4 patients, aged 18 years or above who had clinical diagnosis of FPLD, elevated fasting plasma triglycerides ( $\geq 500 \mathrm{mg} / \mathrm{dL}$ or $\geq 5.7 \mathrm{mmol} / \mathrm{L}$ ), diabetes mellitus associated with lipodystrophy [Hemoglobin $(\mathrm{Hb}) \mathrm{A} 1 \mathrm{c} \geq 7 \%$ and $\leq 12 \%$ ], and hepatic steatosis with mean hepatic fat fraction (HFF) $\geq 6.4 \%$ by magnetic resonance imagining (MRI). Diagnosis of lipodystrophy was based on a deficiency of subcutaneous body fat in a partial fashion assessed by physical examination and low skinfold thickness in the anterior thigh by caliper 
measurement: men $(\leq 10 \mathrm{~mm})$ and women $(\leq 22 \mathrm{~mm})$. Patients were also required to have at least one of the following: genetic diagnosis of FPLD, family history of FPLD or family history of abnormal and similar fat distribution plus one minor criterion or (in the absence of FPLD-associated genetic variant or family history) two minor criteria and body mass index (BMI) $<35 \mathrm{~kg} / \mathrm{m}^{2}$. Minor criteria were defined as follows: 1) requirement for high doses of insulin ( $\geq 200 \mathrm{U} / \mathrm{day}, \geq 2 \mathrm{U} / \mathrm{kg} /$ day), or using U-500 insulin; 2) presence of acanthosis nigricans on physical examination; 3 ) evidence or history of polycystic ovary syndrome (PCOS) or PCOS-like symptoms (hirsutism, oligomenorrhea, and/or polycystic ovaries); 4) history of pancreatitis associated with hypertriglyceridemia; 5) evidence of non-alcoholic fatty liver disease. Patients treated with anti-diabetic (except of GLP-1 agonists), lipid lowering, or atypical antipsychotic medications had to be on stable dose at least 3 months prior to screening. Exclusion criteria included: generalized lipodystrophy or acquired partial lipodystrophy, use of metreleptin or antiobesity drugs within 3 months prior to screening, GLP-1 agonists or systemic corticosteroids or anabolic steroids within 4 weeks prior to screening. Also excluded were patients with estimated glomerular filtration rate (eGFR) $₫ 60 \mathrm{~mL} / \mathrm{min} / 1.73 \mathrm{~m}^{2}$, alanine aminotransferase (ALT) or aspartate aminotransferase (AST) > 2xupper limit of normal (ULN), total bilirubin > ULN, alkaline phosphatase > $1.5 \times U L N$, and platelet count llower limit of normal (LLN).

After a screening period of approximately 6 weeks, which included a 4-week diet stabilization phase, patients were treated with $20 \mathrm{mg}$ of vupanorsen administered subcutaneously every week for 26 weeks, and then followed for 13 additional weeks (post-treatment follow-up period).

The study was conducted at the University of Michigan. Written informed consent was obtained from all participants. The protocol was approved by the Institutional Review Board of Michigan Medicine and complied with the Declaration of Helsinki. The study was registered at ClinicalTrials.gov (NCT03514420).

\section{Study endpoints}

- All efficacy endpoints evaluated the change from baseline at Week 27 (one week after the last dose of the vupanorsen). In addition, an intermediate evaluation of all endpoints was performed at Week 13.

- The primary endpoint was the percent change in fasting triglyceride levels at Week 27. Secondary endpoints included percent change and/or absolute change in: 1) ANGPTL3, and fasting lipids and lipoproteins including very-low density lipoprotein cholesterol (VLDL)-C, total cholesterol (TC), nonHDL-C, LDL-C, apolipoprotein (apo) C-III, apo B, apo B48 and FFA; 2) area under the curve (AUC) in lipid and glycemic parameters including triglycerides, FFA, plasma glucose, serum insulin, and Cpeptide in response to a mixed meal test (MMT);3) HbA1c and insulin resistance assessments including homeostasis model assessment of insulin resistance (HOMA-IR), adipose tissue insulin resistance (ADIPO-IR), insulin sensitivity index (ISI); 4) levels of adiponectin and leptin; 5) hepatic fat fraction (HFF) as assessed by magnetic resonance imaging (MRI); and 6) body fat distribution evaluated by dual-energy X-ray absorptiometry (DEXA).

\section{Measurements}


All laboratory samples (blood and urine) were measured with commercially available assays at MedPace Reference Laboratories (Cincinnati, $\mathrm{OH}$ ). For measurement of lipid parameters, fasted blood samples were collected. LDL-C levels were measured either by ultracentrifugation, or precipitation, and VLDL-C levels were calculated either as TC- (LDL-C + HDL-C), or as triglycerides dived by 5 , respectively. Except for patient 01 who had baseline LDL-C measured by ultracentrifugation and all remaining timepoints by precipitation, all other patients had the same method used for all timepoints. Platelet count measurements were also performed at a local laboratory (Michigan Medicine Clinical Pathology Laboratory).

For the MMT, patients were advised to consume a standardized meal the evening before the test (750 $\mathrm{kcal} ; 20 \%$ of energy from protein, $30 \%$ from fat, and $50 \%$ from carbohydrate) and refrain from consuming alcohol for $72 \mathrm{~h}$. A cannula was inserted into a forearm vein, and after overnight fasting (minimum 8 hours) venous blood sample was taken between 8:00 AM and 10:00 AM. Then, patients consumed a liquid load of Optifast (Optifast; Novartis, Minneapolis, MN; $474 \mathrm{ml}, 320 \mathrm{kcal}, 50 \%$ carbohydrate, 35\% protein, $15 \%$ fat) within a 15-min period. Additional blood samples were taken at 10, 20, 30, 60, 90, 120, $150,180,240$ and 300 min after meal consumption.

Surrogate insulin resistance estimates (HOMA-IR [12] and Adipo-IR [13]) were calculated based on fasting insulin, glucose and FFA concentrations, and ISI [14] was calculated from the blood glucose and insulin levels in the fasting (0 $\mathrm{min})$ and post-meal (120 $\mathrm{min})$ conditions. Insulin resistance indices were measured by utilizing the following equations:

- $\quad$ HOMA $-\mathrm{IR}=($ Fasting glucose $(\mathrm{mmol} / \mathrm{L}) \times$ Fasting insulin $(\mathrm{mU} / \mathrm{L})) / 22.5$

- $\quad$ ADIPO - IR $=$ Fasting FFA $(\mathrm{mmol} / \mathrm{L}) \times$ Fasting insulin $(\mathrm{pmol} / \mathrm{L})$

- $\quad$ ISI $=\log ($ mean insulin $(\mu \mathrm{IU} / \mathrm{L}) / \mathrm{MCR}$; where $\log ($ mean insulin $(\mu \mathrm{IU} / \mathrm{L}))=$ $[(\log$ (fasting insulin $(\mu \mathrm{IU} / \mathrm{L})+\log (2 \mathrm{~h}-$

post prandial insulin $\mu \mathrm{IU} / \mathrm{L}$ )/2》] and MCR (metabolic clearance rate) $=\mathrm{m} /$ mean glucose, $\mathrm{m}=[75000+$ $(($ fasting glucose $(\mathrm{mg} / \mathrm{L})-2 \mathrm{~h}$ post prandial glucose $(\mathrm{mg} / \mathrm{L}) \times 0.19 \times$ weight $(\mathrm{kg})] / 120$ and mean glucose $=$ [fasting glucose $(\mathrm{mmol} / \mathrm{L})+$ glucose at $2 \mathrm{~h}(\mathrm{mmol} / \mathrm{L})] / 2$

Central imaging analysis of MRI data was performed at MedPace Imaging Clinical Laboratory. HFF MRI analysis was performed using scanners with $1.5 \mathrm{~T}$ or a $3 \mathrm{~T}$ field strength and proton density fat fraction (PDFF) acquisition software [15]. Whole-body composition, including fat and lean body masses was evaluated by DEXA (GE Lunar Prodigy, model PA + 41744) [16].

\section{Safety monitoring}

In addition to standard safety monitoring, monitoring and stopping rules for platelet count, liver and renal function were prespecified in the protocol. The platelet count was monitored every 2 weeks and analyzed 
simultaneously by both central and local laboratories, while liver and renal function tests were monitored every 2 weeks during the first 3 months of the treatment period and monthly thereafter. Per protocol, the investigator could interrupt or permanently discontinue study treatment for any safety reason, including clinically meaningful changes in any clinical laboratory results.

\section{Statistical Analysis}

For this small, single-arm proof of concept study, descriptive statistics were used to summarize the observed and change from baseline data. The AUC of lipid and glycemic parameters in response to MMT was calculated from the curves obtained from the values plotted over time using the linear trapezoidal method.

\section{Results}

\section{Patient Population}

A total of 8 patients with FPLD were screened, and 4 patients were enrolled and treated with vupanorsen for 26 weeks. The reasons for screen failure were: diagnosis of diabetes mellitus made less than 6 months from screening (2 patients), an estimated glomerular filtration rate lower than 60 $\mathrm{mL} / \mathrm{min} / 1.73 \mathrm{~m}^{2}$, and liver function tests $>2$ times ULN. All enrolled patients completed the study.

Demographic and baseline characteristics for each patient are presented in Table 1. The mean age was 42.2 \pm 3.7 years (range: $38-47$ years), and 3 patients were females. Genetic confirmation of FPLD was obtained from 2 patients, showing different $L M N A$ gene mutations. All 4 patients had family history of abnormal and similar fat distribution. Baseline laboratory values are presented in Table 2 and Table 4. Baseline mean \pm SD triglyceride level was $9.24 \pm 4.9 \mathrm{mmol} / \mathrm{L}(817.8 \pm 431.9 \mathrm{mg} / \mathrm{dL})$, and, except for HDL$\mathrm{C}$, all other mean baseline lipid and lipoprotein parameters were elevated. By protocol design, patients also had elevated $\mathrm{HbA} 1 \mathrm{c}(9.5 \pm 1.3 \%)$, and HFF (19.7 $\pm 9.6 \%)$. Baseline leptin and adiponectin levels (shown in Table 1) were within range expected from the underlying diagnoses of FPLD.

Change from baseline in fasting triglycerides, ANGPTL3, and other lipids and lipoproteins with treatment.

Treatment with vupanorsen resulted in lowering of serum ANGPTL3 by a mean of $54.7 \%$ (Table 2). This result was accompanied by a mean reduction in levels of fasting triglycerides (by $59.9 \%$ ), VLDL-C (by 53.5\%), non-HDL-C (by 20.9\%), apoC-III (by 50.9\%), apoB48 (by $69.2 \%$ ), and FFA (by $41.7 \%$ ). Reductions in these parameters were already observed at Week 13 in all patients (Fig. 1). LDL-C levels showed a small increase, and no effect of vupanorsen was observed on apoB, and HDL-C (Table 2).

Effect of vupanorsen treatment on fasting plasma glucose, $\mathrm{HbA1c}$, and insulin resistance indices.

Fasting plasma glucose (FPG) showed a decrease from baseline by $3.2 \pm 0.7 \mathrm{mmol} / \mathrm{L}$ at Week 27 and there was no significant effect on HbA1c levels (Table 3). Treatment with vupanorsen reduced the adipose tissue insulin resistance as measured by ADIPO-IR by $55 \%$ while HOMA-IR and ISI showed no 
change (Table 3, Fig. 2A). However, at baseline, the HOMA-IR values were inversely associated with serum FFA (Fig. 2B). One participant (subject $n^{\circ} 08$ ) had a very unstable and difficult to control diabetes, requiring treatment with very high doses of U500 insulin, and was excluded from analyses of FPG and insulin-level parameters.

Change in postprandial lipid and glucose metabolism as measured during the mixed meal test with vupanorsen

At baseline, triglyceride levels did not rise post-meal; and in fact, they were slightly reduced in 3 out of 4 patients (Additional file 1). Following treatment with vupanorsen, the MMT performed at Week 27 showed decreases in AUC for postprandial triglyceride and FFA levels compared to baseline by $60 \%$ and $32 \%$, respectively (Table 4). The individual values of AUC for triglyceride levels (Fig. 3A) show a consistent effect of vupanorsen observed in all patients with a progressive reduction in the AUCs with treatment over time. Similar pattern was observed for FFA in 3 out of 4 patients (Fig. 3B). Reduction in mean post meal triglyceride levels before and after vupanorsen is shown in Fig. 3C. Small decreases in mean AUCs for glucose and C-peptide were observed, while insulin AUCs showed a trend toward an increase (Table 4, Additional file 1).

\section{Changes in HFF, body fat distribution and fat cell hormones}

The mean HFF displayed a specific pattern during vupanorsen treatment with an increase from baseline of $19.7 \pm 9.6 \%$ (mean \pm SD) to $25.1 \pm 8.7 \%$ (absolute increase from baseline by $5.4 \pm 4.4 \%$ ) at Week 13 , followed by a subsequent decrease to $18.5 \pm 9.5 \%$ (absolute decrease from baseline by $-1.1 \pm 1.2 \%$ ) at Week 27. This trend was observed in all 4 treated patients (Fig. 4A).

There was no change at Week 27 in the amount of fat in the trunk or total body, however there was a trend towards an increase in the extremity fat (Fig. $4 \mathrm{~B}$ and $\mathrm{C}$ ). The ratio of fat mass in the arms to total body fat increased by $15.6 \pm 18.8 \%$ while the ratio of the fat mass in the legs to total body fat also showed a numeric increase by $6.2 \pm 6.4 \%$. Other body composition parameters remained unchanged throughout the treatment period including body weight and BMI. No effect was observed on leptin or adiponectin levels with treatment (Additional file 2).

\section{Safety and tolerability}

The 4 patients reported a total of 90 adverse events (AEs) during the study, among them 70 AEs $(77.8 \%)$ were considered mild in severity, and $18(20 \%)$ were considered as related to the drug with the majority related to hepatic steatosis and liver enzyme increases (discussed below). Three patients experienced a total of 8 serious adverse events (SAEs), all assessed as not related to the study drug and recovered. These were: ventricular tachycardia and angina pectoris that occurred during and following elective dobutamine stress test in a patient with a history of chest pain, palpitations, and family history of premature coronary artery disease (CAD); acute pancreatitis in a patient with a history of pancreatitis: atrial flutter, atrial fibrillation with either troponin increase or pulmonary edema, respectively, and acute coronary syndrome in a patient with diffuse 3 vessel CAD not amendable to revascularization. No patient 
discontinued treatment due to $A E, 3$ patients had short interruptions of the study drug either due to $S A E s$ (2 patients), or, in case of the first patient enrolled, due to $A E$ of hepatic steatosis with transient elevation of HFF that was an unexpected finding at the time it was observed. Treatment was resumed after an assessment by a hepatologist, and despite treatment continuation, HFF returned to baseline values. Overall, transient increases in transaminases to $>2$ and $<3$ times ULN (or $<2$ times baseline value in a patient with elevated baseline) were observed in 3 patients early during the study with peak values at Week 4-13 and return to normal values thereafter despite continuation of treatment. These increases in transaminases seemed to accompany increases in HFF and were not associated with changes in bilirubin or INR levels.

One patient reported mild injection site reaction (dryness), and there were no reports of flu-like reactions. There was no effect of vupanorsen on platelet count (no platelet value below LLN), and no clinically significant changes in renal function tests.

\section{Discussion}

In this proof-of-concept study in patients with genetic or clinical diagnosis of FPLD, treatment with the novel therapeutic vupanorsen targeting ANGPTL3, resulted in a robust reduction in fasting triglycerides associated with a reduction in ANGPTL3, VLDL-C, non-HDL-C, and apo C-III levels. These effects were similar to those reported for vupanorsen in patients with diabetes, hepatic steatosis and hypertriglyceridemia [17]. Reductions in apoB48, postprandial triglyceride, fasting glucose, fasting and postprandial FFA levels, and ADIPO-IR index were also observed. In addition, changes in HFF and DEXA parameters suggested dynamic changes in fat partitioning.

Although initial presentation of FPLD is very heterogeneous, the diagnosis of FPLD can be established with a careful clinical assessment of fat distribution through visual and physical examination [18]. In addition, whole body DEXA has been utilized to accurately visualize and document the fat tissue distribution pattern [19]. Genetic testing, when available, can confirm the diagnosis of FPLD [20]. In our study, two patients were classified as FPLD2 by a genetic confirmation of mutation on the LMNA gene, while the other two patients were classified as FPLD1, because no causative single genetic variants could be identified in known lipodystrophy genes. As expected, patients in the two subgroups presented with different amounts of adiposity. FPLD1 patients had more fat mass than FPLD2, but all patients had upper-body predominant truncal distribution with patients with FPLD1 having more abdominal adiposity. Regardless of the etiology and the differing amount of residual fat mass, all patients in this study presented with the triad of insulin resistance with clinical diabetes, significant dyslipidemia, and fatty liver [21], and uniformly responded to vupanorsen with lowering of triglyceride, and TRL levels, as well as ADIPO-IR index.

Hypertriglyceridemia is an important metabolic problem in patients with FPLD. The causative mechanism for the elevation of serum triglycerides in FPLD is still unknown, but it may be related to an increase in energy intake, and ectopic lipid deposition particularly in the liver, leading to enhanced VLDL secretion 
$[22,23]$. In addition, this condition can be viewed as an exaggerated form of metabolic syndrome as the increase in upper and central depots are relatively more exaggerated when the lower extremity fat is absent. We have recently published that metabolic burden of the patients with FPLD are equivalent to those individuals who have BMI at least 7 to 10 points higher [24]. In addition, a recent paper from Brown and colleagues demonstrates a markedly increased hepatic de novo lipogenesis [25].

Hypertriglyceridemia may contribute to a high prevalence of cardiac disease among patients with FPLD [26] and reports of early cardiac mortality in this patient population [27]. However limited, our study showed that targeting ANGPTL3 with vupanorsen can reduce triglyceride (fasting and postprandial) and TRL levels. For this reason, it may become a possible option for the management of FPLD. In addition, FPLD patients are also at increased risk for acute pancreatitis, making effective treatment of hypertriglyceridemia an urgent priority.

Interestingly, the ADIPO-IR was reduced by $55 \%$ from baseline after vupanorsen treatment, indicating a reduction in insulin resistance of the total adipose tissue compartment. ADIPO-IR is calculated from a single measurement of FFAs and insulin concentrations [28], thus our results likely reflect the changes in circulating FFAs after vupanorsen treatment. It has been debated why, or even if, the FFAs are elevated in FPLD (and in other human lipodystrophy syndromes) because of the limited fat compartments and the expectation that patients cannot have increased lipolysis in the fasting state [29]. It has also been questioned whether the elevated FFAs are a measurement artefact due to in vitro breakdown of the triglycerides via the white blood cells. In this study, samples were kept on ice and processed rapidly to minimize potential deterioration. In addition, we have previously reported increased in vivolipolysis even in patients with generalized lipodystrophy [29]. This raises the possibility that lipolysis can occur in alternative tissues via ectopic lipases to compensate for reduced lipolysis in the adipocyte compartment. However, more direct data are required to determine the source of increased lipolysis and FFA levels in individuals with FPLD. The ADIPO-IR has been reported to correlate with hepatic fat content, a marker of ectopic fat deposition [30], and reduction in the index is usually coupled with improvements in nonalcoholic steatohepatitis histopathology in more common settings [30]. Although there was a transient increase in HFF at Week 13 in this study, these levels returned to baseline at Week 27, despite continued treatment with vupanorsen. Consistent with the observation that ADIPO-IR is a measure independent of the HOMA-IR index for glucose metabolism [31], there was no change in HOMA-IR after treatment with vupanorsen, suggesting that the effect of the drug is not on a pathway that is downstream of Glut-4 translocation and glucose utilization for the whole body, but more related to changes in lipid storage and mobilization.

Treatment with vupanorsen was also associated with reduced AUCs of triglyceride, FFA, and glucose levels in response to MMT. The observation of lowering in post meal triglyceride levels in almost all patients is consistent with the observation that carriers of homozygous ANGPTL3 loss-of-function mutation show essentially no postprandial increase in TRL in response to a fat challenge [32]. It is also interesting to note that patients with FPLD had more pronounced abnormalities in the fasting state compared to the abnormalities observed in their post-absorptive state, highlighting and supporting the 
importance of hepatic de novo lipogenesis as an important contributor to the dyslipidemia associated with lipodystrophy.

Overall adiposity was stable after treatment, but careful analyses of the DEXA parameters and liver fat suggest that there may be dynamic changes in lipid partitioning between compartments. The heterogeneous nature of adipose tissue and various roles for the cells that compose this tissue in its various depots throughout the body have been reported [33,34]. The early increase and further return to baseline levels of HFF may suggest mobilization of lipid and a shift in partitioning; however, further studies with animal models or in vitro models such as 3D cell culture [35] are needed to better understand the changes in fat distribution observed in our study.

In this study, there was a change in fasting glucose levels at week 27 with only a small decrease in $\mathrm{HbA} 1 \mathrm{c}$. We can speculate that the improvement in fasting glucose is an indirect effect secondary to changes in lipid partitioning and reduction on hepatic de novo lipogenesis. Since these are indirect effects and depend on certain changes in lipid dynamics to occur first, one can postulate that if the patients were to be followed longer, the $\mathrm{HbA} 1 \mathrm{c}$ could have been lowered to a greater extent. Longer studies can shed further light and help to provide support for these initial hypotheses generated by our data.

The only drug that has been approved for treatment of lipodystrophy is metreleptin, a recombinant analogue of human leptin used as leptin replacement therapy to treat the metabolic complications of lipodystrophy. Metreleptin is approved only for the treatment of generalized lipodystrophy and not for FPLD in the US, even though there have been reports of benefit in some FPLD patients with relatively low leptin levels [36]. In the EU, metreleptin is approved for the treatment of FPLD in adults and children above the age of 12 years, but only when standard treatments have failed [37]. Interestingly, metreleptin has also been shown to reduce elevated ANGPTL3 levels observed in generalized lipodystrophy, however this effect was relatively small ( $20 \%$ reduction) and not correlated with changes in triglycerides or glycemia, hence probably not responsible for the overall metabolic benefits of metreleptin [38]. It is important to note that the FPLD patients in the present study had baseline circulating leptin and adiponectin levels that were concurrent with previously published levels depending on the subtype of FPLD. No change in leptin or adiponectin levels was observed upon treatment with vupanorsen, suggesting that the endocrine function of the adipocytes was not impacted by the drug.

The number of AEs observed in this study is consistent with diverse pathologies and disease burden in patients with FPLD, and this reflects the common serious metabolic and organ specific manifestations of the disease. The open-label nature of the study and the lack of a control group limits the conclusions that can be reached on safety and a large placebo-controlled study of vupanorsen in patients with dyslipidemia is ongoing (NCT 04516291). Treatment with vupanorsen was well tolerated and not associated with any changes in platelet count consistent with the overall experience with GaINAc3 ASOs [39]. 
Limitations of this work include the open-label design, lack of a control group, and the small number of patients treated. In addition, only one dose was studied, and it is possible that larger pharmacodynamic effects may have been reached with a dose achieving a greater amount of ANGPTL3 knockdown. However, these limitations are inherent to the nature of drug development at this early stage in a rare disease population.

\section{Conclusion}

The results of this study suggest that targeting of ANGPTL3 with vupanorsen could address several metabolic abnormalities in patients with FPLD. This study also raises key questions on the importance of lipid partitioning for human metabolism.

\section{Abbreviations}




\begin{tabular}{ll} 
ADIPO-IR & Adipose tissue insulin resistance \\
\hline ANGPTL3 & Angiopoietin-like protein 3 \\
\hline Apo & Apolipoprotein \\
\hline AUC & Area under the curve \\
\hline BMI & Body mass index \\
\hline DEXA & Dual-energy X-ray absorptiometry \\
\hline FFA & Free fatty acid \\
\hline FPLD & Familial partial lipodystrophy \\
\hline GaINAc3 & N-acetyl galactosamine-3 \\
\hline Hb & Hemoglobin \\
\hline HDL-C & High-density lipoprotein cholesterol \\
\hline HOMA-IR & Homeostasis model assessment of insulin resistance \\
\hline LDL-C & Low-density lipoprotein cholesterol \\
\hline LLN & Lower limit of normal \\
\hline MRI & Magnetic resonance imagining \\
\hline Non-HDL-C & non-high-density lipoprotein cholesterol \\
\hline T2DM & Type 2 diabetes mellitus \\
\hline TC & Total cholesterol \\
\hline TRL & Triglyceride rich lipoprotein \\
\hline ULN & Upper limit of normal \\
\hline VLDL-C & Very-low-density lipoprotein cholesterol \\
\hline
\end{tabular}

\section{References}

1. Foss-Freitas M, Akinci B, Lou Y, Stratton A, Oral E. Diagnostic strategies and clinical management of lipodystrophy. 2020.

2. Bagias C, Xiarchou A, Bargiota A, Tigas S. Familial Partial Lipodystrophy (FPLD): Recent Insights. Diabetes Metab Syndr Obes. 2020;13:1531-44. 
3. Oral EA, Gorden P, Cochran E, Araujo-Vilar D, Savage DB, Long A, Fine G, Salinardi T, Brown RJ. Longterm effectiveness and safety of metreleptin in the treatment of patients with partial lipodystrophy. Endocrine. 2019;64:500-11.

4. Polyzos SA, Perakakis N, Mantzoros CS. Fatty liver in lipodystrophy: A review with a focus on therapeutic perspectives of adiponectin and/or leptin replacement. Metabolism. 2019;96:66-82.

5. Ajluni N, Meral R, Neidert AH, Brady GF, Buras E, McKenna B, DiPaola F, Chenevert TL, Horowitz JF, Buggs-Saxton $\mathrm{C}$, et al. Spectrum of disease associated with partial lipodystrophy: lessons from a trial cohort. Clin Endocrinol (Oxf). 2017;86:698-707.

6. Akinci B, Oral EA, Neidert A, Rus D, Cheng WY, Thompson-Leduc P, Cheung HC, Bradt P, Foss de Freitas MC, Montenegro RM, et al. Comorbidities and Survival in Patients With Lipodystrophy: An International Chart Review Study. J Clin Endocrinol Metab. 2019;104:5120-35.

7. Kersten S. Angiopoietin-like 3 in lipoprotein metabolism. Nat Rev Endocrinol. 2017;13:731-9.

8. Reeskamp LF, Tromp TR, Stroes ESG. The next generation of triglyceride-lowering drugs: will reducing apolipoprotein C-III or angiopoietin like protein 3 reduce cardiovascular disease? Curr Opin Lipidol. 2020;31:140-6.

9. Dewey FE, Gusarova V, Dunbar RL, O'Dushlaine C, Schurmann C, Gottesman O, McCarthy S, Van Hout CV, Bruse S, Dansky HM, et al. Genetic and Pharmacologic Inactivation of ANGPTL3 and Cardiovascular Disease. N Engl J Med. 2017;377:211-21.

10. Christopoulou E, Elisaf M, Filippatos T. Effects of Angiopoietin-Like 3 on Triglyceride Regulation, Glucose Homeostasis, and Diabetes. Dis Markers. 2019;2019:6578327.

11. Graham MJ, Lee RG, Brandt TA, Tai LJ, Fu W, Peralta R, Yu R, Hurh E, Paz E, McEvoy BW, et al. Cardiovascular and Metabolic Effects of ANGPTL3 Antisense Oligonucleotides. N Engl J Med. 2017;377:222-32.

12. Matthews DR, Hosker JP, Rudenski AS, Naylor BA, Treacher DF, Turner RC. Homeostasis model assessment: insulin resistance and beta-cell function from fasting plasma glucose and insulin concentrations in man. Diabetologia. 1985;28:412-9.

13. Gastaldelli A, Gaggini M, DeFronzo RA. Role of Adipose Tissue Insulin Resistance in the Natural History of Type 2 Diabetes: Results From the San Antonio Metabolism Study. Diabetes. 2017;66:815-22.

14. Ghosh C, Mukhopadhyay P, Ghosh S, Pradhan M. Insulin sensitivity index (ISIO, 120) potentially linked to carbon isotopes of breath CO2 for pre-diabetes and type 2 diabetes. Sci Rep. 2015;5:11959.

15. Idilman IS, Aniktar H, Idilman R, Kabacam G, Savas B, Elhan A, Celik A, Bahar K, Karcaaltincaba M. Hepatic steatosis: quantification by proton density fat fraction with MR imaging versus liver biopsy. Radiology. 2013;267:767-75.

16. Valerio CM, Godoy-Matos A, Moreira RO, Carraro L, Guedes EP, Moises RS, Mory PB, de Souza LL, Russo LA, Melazzi AC. Dual-energy X-ray absorptiometry study of body composition in patients with lipodystrophy. Diabetes Care. 2007;30:1857-9. 
17. Gaudet D, Karwatowska-Prokopczuk E, Baum SJ, Hurh E, Kingsbury J, Bartlett VJ, Figueroa AL, Piscitelli P, Singleton W, Witztum JL, et al. Vupanorsen, an $\mathrm{N}$-acetyl galactosamine-conjugated antisense drug to ANGPTL3 mRNA, lowers triglycerides and atherogenic lipoproteins in patients with diabetes, hepatic steatosis, and hypertriglyceridaemia. Eur Heart J. 2020;41:3936-45.

18. Brown RJ, Araujo-Vilar D, Cheung PT, Dunger D, Garg A, Jack M, Mungai L, Oral EA, Patni N, Rother KI, et al. The Diagnosis and Management of Lipodystrophy Syndromes: A Multi-Society Practice Guideline. J Clin Endocrinol Metab. 2016;101:4500-11.

19. Meral R, Ryan BJ, Malandrino N, Jalal A, Neidert AH, Muniyappa R, Akinci B, Horowitz JF, Brown RJ, Oral EA. "Fat Shadows" From DXA for the Qualitative Assessment of Lipodystrophy: When a Picture Is Worth a Thousand Numbers. Diabetes Care. 2018;41:2255-8.

20. Garg A. Clinical review\#: Lipodystrophies: genetic and acquired body fat disorders. J Clin Endocrinol Metab. 2011;96:3313-25.

21. Huang-Doran I, Sleigh A, Rochford JJ, O'Rahilly S, Savage DB. Lipodystrophy: metabolic insights from a rare disorder. J Endocrinol. 2010;207:245-55.

22. Joseph J, Shamburek RD, Cochran EK, Gorden P, Brown RJ. Lipid regulation in lipodystrophy versus the obesity-associated metabolic syndrome: the dissociation of HDL-C and triglycerides. J Clin Endocrinol Metab. 2014;99:E1676-80.

23. Akinci B, Meral R, Oral EA. Phenotypic and Genetic Characteristics of Lipodystrophy: Pathophysiology, Metabolic Abnormalities, and Comorbidities. Curr Diab Rep. 2018;18:143.

24. Koo E, Foss-Freitas MC, Meral R, Ozer M, Eldin AJ, Akinci B, Miller N, Rothberg AE, Oral EA. The Metabolic Equivalent BMI in Patients with Familial Partial Lipodystrophy (FPLD) Compared with Those with Severe Obesity. Obesity (Silver Spring). 2021;29:274-8.

25. Baykal AP, Parks EJ, Shamburek R, Syed-Abdul MM, Chacko S, Cochran E, Startzell M, Gharib AM, Ouwerkerk R, Abd-Elmoniem KZ, et al: Leptin decreases de novo lipogenesis in patients with lipodystrophy. JCl Insight 2020, 5.

26. Jalal Eldin A, Akinci B, Monteiro da Rocha A, Meral R, Yildirim Simsir I, Adiyaman SC, Ozpelit E, Bhave $\mathrm{N}$, Gen R, Yurekli B, et al: Cardiac Phenotype in Familial Partial Lipodystrophy. Clinical endocrinology 2021.

27. Akinci B, Onay H, Demir T, Savas-Erdeve S, Gen R, Simsir IY, Keskin FE, Erturk MS, Uzum AK, Yaylali GF, et al. Clinical presentations, metabolic abnormalities and end-organ complications in patients with familial partial lipodystrophy. Metab Clin Exp. 2017;72:109-19.

28. Gastaldelli A, Cusi K, Pettiti M, Hardies J, Miyazaki Y, Berria R, Buzzigoli E, Sironi AM, Cersosimo E, Ferrannini E, Defronzo RA. Relationship between hepatic/visceral fat and hepatic insulin resistance in nondiabetic and type 2 diabetic subjects. Gastroenterology. 2007;133:496-506.

29. Gastaldelli A, Harrison SA, Belfort-Aguilar R, Hardies LJ, Balas B, Schenker S, Cusi K. Importance of changes in adipose tissue insulin resistance to histological response during thiazolidinedione treatment of patients with nonalcoholic steatohepatitis. Hepatology. 2009;50:1087-93. 
30. Bell LN, Wang J, Muralidharan S, Chalasani S, Fullenkamp AM, Wilson LA, Sanyal AJ, Kowdley KV, Neuschwander-Tetri BA, Brunt EM, et al. Relationship between adipose tissue insulin resistance and liver histology in nonalcoholic steatohepatitis: a pioglitazone versus vitamin $E$ versus placebo for the treatment of nondiabetic patients with nonalcoholic steatohepatitis trial follow-up study. Hepatology. 2012;56:1311-8.

31. Sondergaard E, Espinosa De Ycaza AE, Morgan-Bathke M, Jensen MD. How to Measure Adipose Tissue Insulin Sensitivity. J Clin Endocrinol Metab. 2017;102:1193-9.

32. Tikkanen E, Minicocci I, Hallfors J, Di Costanzo A, D'Erasmo L, Poggiogalle E, Donini LM, Wurtz P, Jauhiainen M, Olkkonen VM, Arca M. Metabolomic Signature of Angiopoietin-Like Protein 3 Deficiency in Fasting and Postprandial State. Arterioscler Thromb Vasc Biol. 2019;39:665-74.

33. Cohen P, Spiegelman BM. Cell biology of fat storage. Mol Biol Cell. 2016;27:2523-7.

34. Lynes MD, Tseng YH. Deciphering adipose tissue heterogeneity. Ann N Y Acad Sci. 2018;1411:5-20.

35. Bender R, McCarthy M, Brown T, Bukowska J, Smith S, Abbott RD, Kaplan DL, Williams C, Wade JW, Alarcon A, et al. Human Adipose Derived Cells in Two- and Three-Dimensional Cultures: Functional Validation of an In Vitro Fat Construct. Stem Cells Int. 2020;2020:4242130.

36. Sekizkardes H, Cochran E, Malandrino N, Garg A, Brown RJ. Efficacy of Metreleptin Treatment in Familial Partial Lipodystrophy Due to PPARG vs LMNA Pathogenic Variants. J Clin Endocrinol Metab. 2019;104:3068-76.

37. Akinci B, Meral R, Oral EA. Update on Therapeutic Options in Lipodystrophy. Curr Diabetes Rep. 2018;18:139.

38. Muniyappa R, Abel BS, Asthana A, Walter MF, Cochran EK, Remaley AT, Skarulis MC, Gorden P, Brown RJ. Metreleptin therapy lowers plasma angiopoietin-like protein 3 in patients with generalized lipodystrophy. J Clin Lipidol. 2017;11:543-50.

39. Crooke ST, Baker BF, Xia S, Yu RZ, Viney NJ, Wang Y, Tsimikas S, Geary RS. Integrated Assessment of the Clinical Performance of GalNAc3-Conjugated 2'-O-Methoxyethyl Chimeric Antisense Oligonucleotides: I. Human Volunteer Experience. Nucleic Acid Ther. 2019;29:16-32.

\section{Figures}


A)

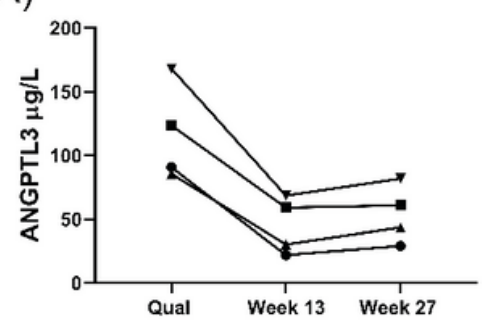

B)

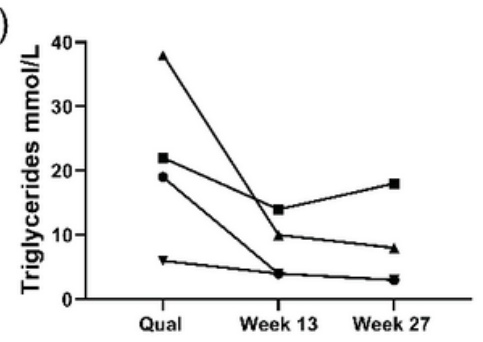

C)

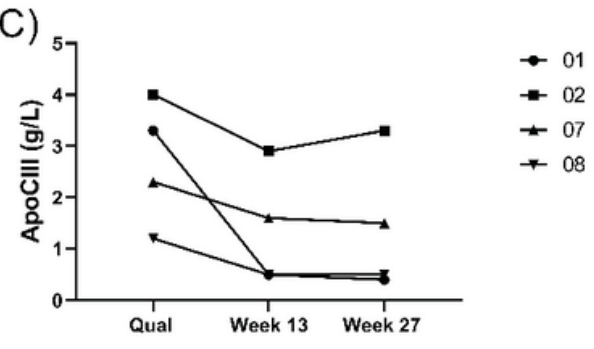

\section{Figure 1}

Effect of vupanorsen on ANGPTL3 (A), Triglyceride (B), and VLDL-C (C) levels in individual participants over the course of the study. Legend shows the subject numbers to link individual data from different figures.

A)

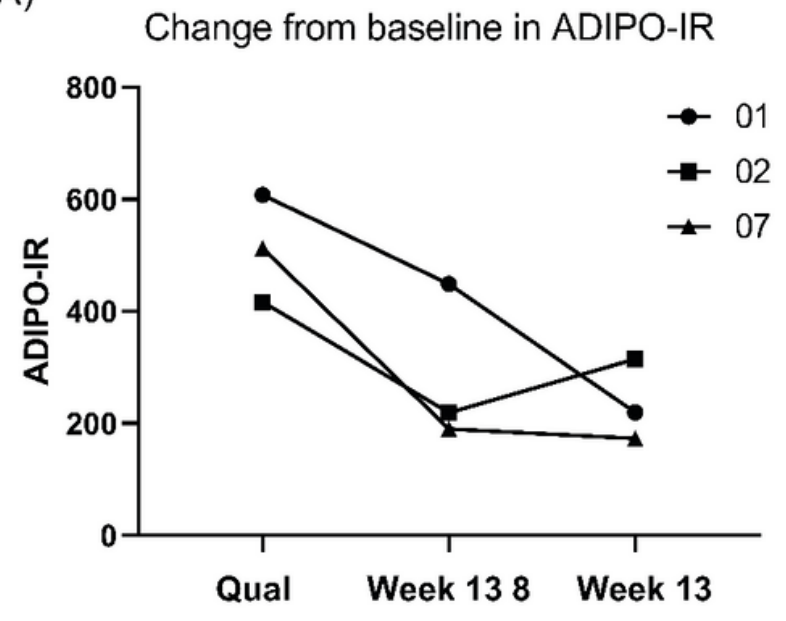

B)
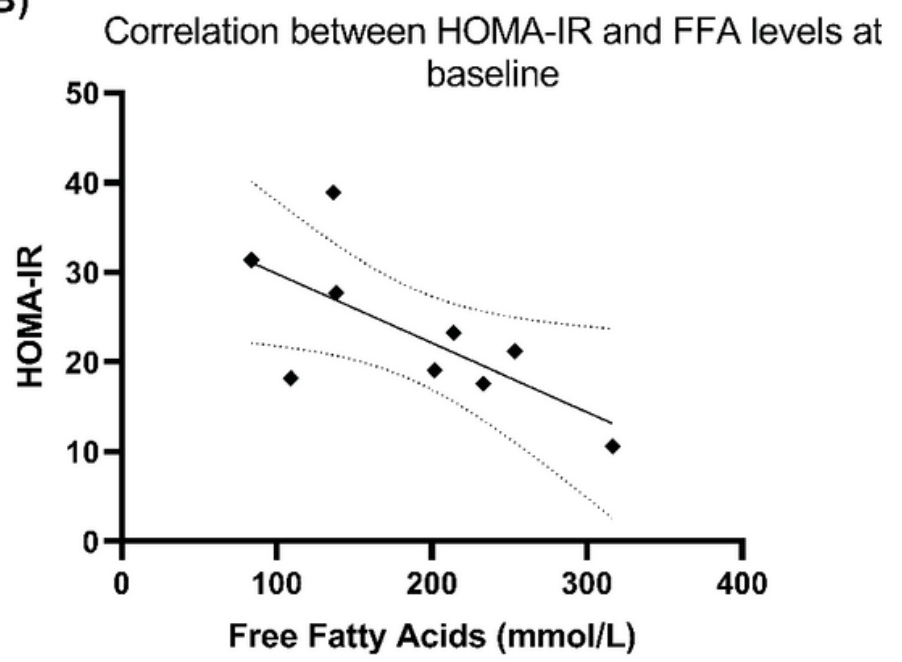

Figure 2

Change in insulin resistance indices during the study. A) Adipo-IR (adipose tissue insulin resistance index) and B) correlation between HOMA-IR (homeostatic model assessment- insulin resistance index) and free fatty acids. Legend shows the subject numbers to link individual data from different figures. 


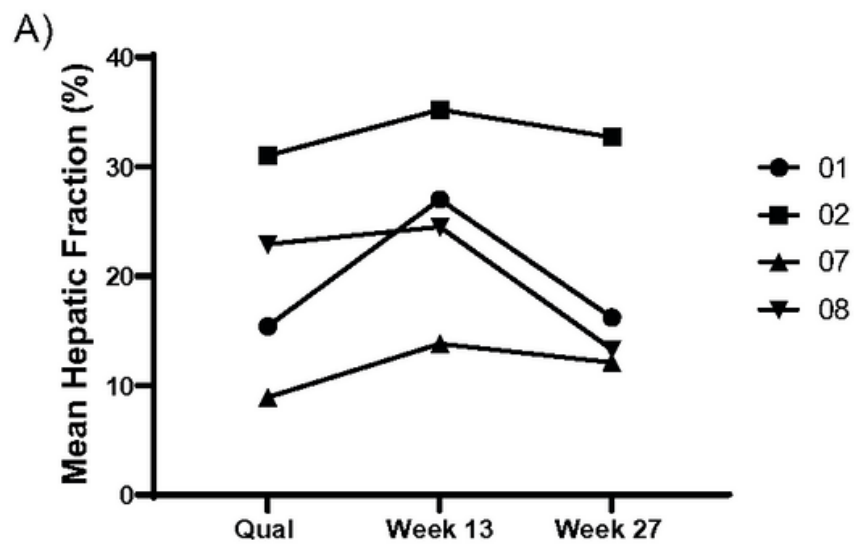

B)

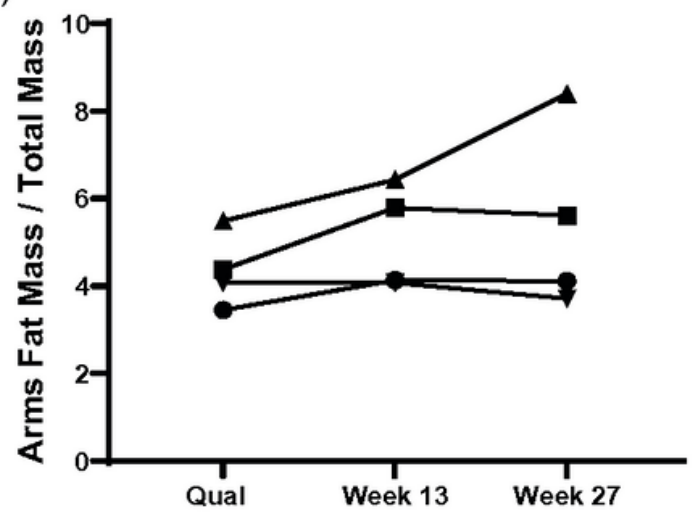

C)

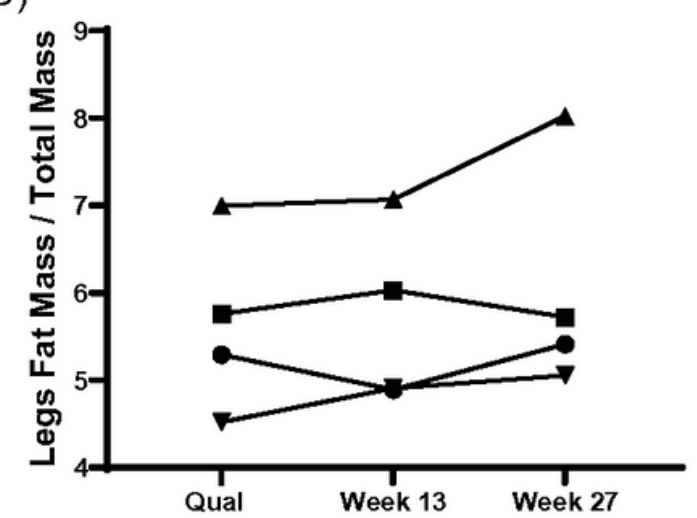

Figure 3

Postprandial response in triglyceride (A) and free fatty acid (FFA) (B) area under the curve (AUC) in the individual subjects, and mean triglyceride levels from all participants (C) during the mixed meal test at key study time points. Legend shows the subject numbers to link individual data from different figures. Conversion factor for SI units for triglycerides $=0.0113(\mathrm{mmol} / \mathrm{L})$ 
A)

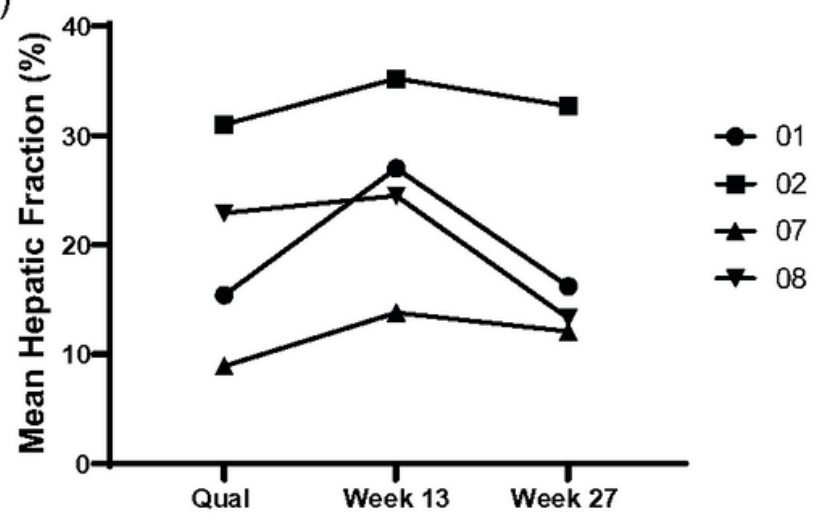

B)

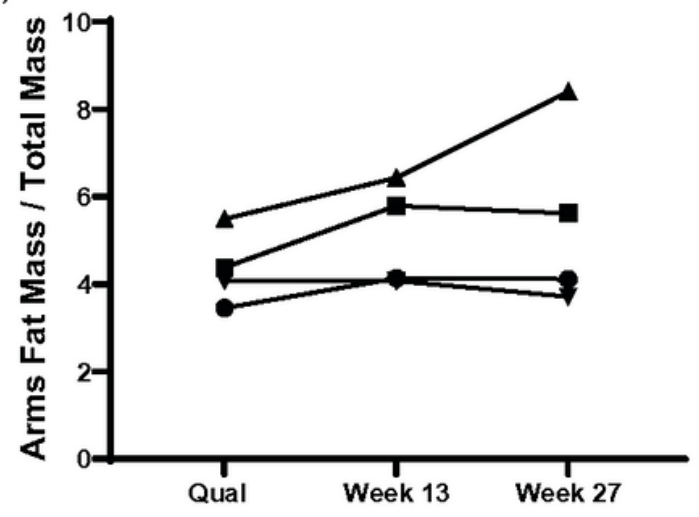

C)

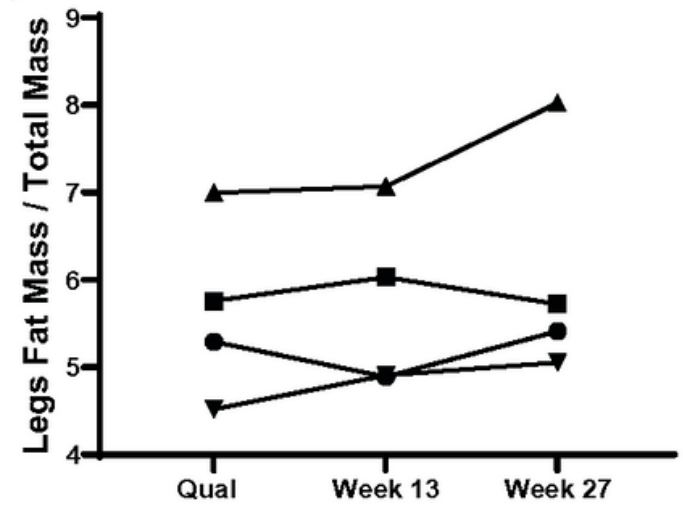

\section{Figure 4}

Change in hepatic fat fraction ( $A$ ) and body fat distribution (B and $C$ ) from baseline during the study in individual subjects. Legend shows the subject numbers to link individual data from different figures.

\section{Supplementary Files}

This is a list of supplementary files associated with this preprint. Click to download.

- Adfile1TIF.pdf

- Adfile2TIF.pdf 